This item was submitted to Loughborough's Research Repository by the author.

Items in Figshare are protected by copyright, with all rights reserved, unless otherwise indicated.

\title{
A CMOE-CMA RAKE receiver structure for near-far frequency selective fading CDMA channels
}

PLEASE CITE THE PUBLISHED VERSION

PUBLISHER

(c) IEEE

VERSION

VoR (Version of Record)

LICENCE

CC BY-NC-ND 4.0

\section{REPOSITORY RECORD}

Yuvapoositanon, Peerapol, and Jonathon Chambers. 2019. "A CMOE-CMA RAKE Receiver Structure for Near-far Frequency Selective Fading CDMA Channels”. figshare. https://hdl.handle.net/2134/5781. 
This item was submitted to Loughborough's Institutional Repository (https://dspace.lboro.ac.uk/) by the author and is made available under the following Creative Commons Licence conditions.

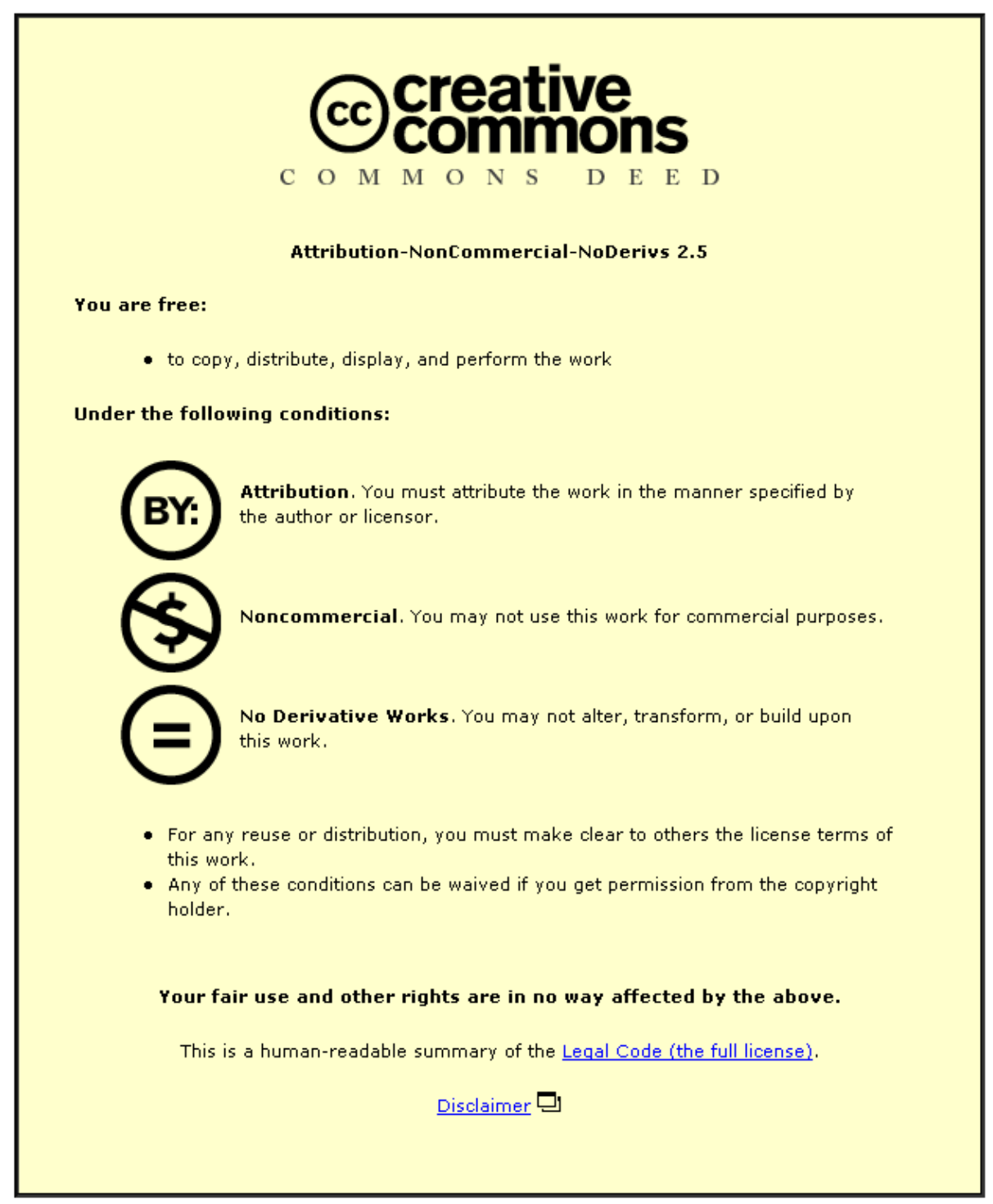

For the full text of this licence, please go to: http://creativecommons.org/licenses/by-nc-nd/2.5/ 


\title{
A CMOE-CMA RAKE RECEIVER STRUCTURE FOR NEAR-FAR FREQUENCY-SELECTIVE FADING CDMA CHANNELS
}

\author{
Peerapol Yuvapoositanon and Jonathon A. Chambers \\ Signal and Image Processing Group, \\ Department of and Electronic and Electrical Engineering, \\ University of Bath, \\ Bath BA2 7AY, United Kingdom \\ Email: eeppay@bath.ac.uk
}

\begin{abstract}
A novel initialization scheme for a constant modulus algorithm RAKE (CMA-RAKE) receiver for frequency-selective fading asynchronous code-division multiple access (CDMA) channels is proposed. The solutions from the minimization of the constrained minimum output energy (CMOE) criterion are adopted as the initialization of the subsequent CMA receivers. The proposed receiver is proven to be near-far resistant at different levels of nearfar problems. Simulations confirm the superiority of the receiver over the existing RAKE receivers in terms of signal-to-interference plus noise (SINR) ratio over a wide range of near-far situations.
\end{abstract}

\section{INTRODUCTION}

In wideband CDMA systems, a RAKE receiver is used to gather signal energies at different chip delays by coherently combining the outputs from a bank of correlators or arms. However, the conventional RAKE receiver is susceptible to the near-far probiem: the situation where interfering users are stronger than the desired user. In [1], the conventional RAKE structure is maintained but the correlators are replaced by the near-far resistant constrained minimum output energy (CMOE) detectors developed in [2]. This receiver is called the decorrelating RAKE (D-RAKE) receiver due to the decorrelating nature of the CMOE detectors. The performance in terms of the mean-square error (MSE) of the D-RAKE receiver is shown to be superior to that of the single-arm $\mathrm{CMOE}$ detector [2].

The idea of replacing the correlators in the conventional RAKE receiver with adaptive CMA equalizers is proposed in [3] by assuming knowledge of the correct timing of the desired user. After convergence, the MSE performance of the CMA-RAKE receiver is shown to be better than that of the D-RAKE receiver [1]. However, the CMA-RAKE receiver (and any receiver using the constant modulus stochastic gradient (CM-SG) criterion) is susceptible to misconvergence in the near-far situation due to its multimodal cost surface. Unless a prewhitening process is employed, the solution is likely to be dominated by the stronger interfering solutions and results in failure to successfully retrieve the desired transmissions [4].

In this paper, we propose an initialization scheme for the CMARAKE receiver by using the solution from the minimization of the

This work was supported by the Mahanakorn University of Technology, Bangkok, Thailand.
CMOE criterion (CMOE-CMA RAKE) for frequency-selective fading asynchronous CDMA channels. The signal-to-interference plus noise ratio (SINR) is used as a performance measure for the desired solution. It is shown from the simulations that for different levels of near-far problems, the SINR performance of the CMOECMA RAKE receiver is improved upon that of the D-RAKE [1] and CMA-RAKE receivers [3]. The SINR of the CMOE-CMA RAKE receiver is shown to be maintained for the different levels of near-far situations.

\section{SYSTEM MODEL}

For the real system model from Honig et al. [5], the baseband received signal for a $K$-user asynchronous CDMA channel is defined as

$$
r(t)=\sum_{i=-\infty}^{\infty} \sum_{k=1}^{K} A_{k} b_{k}[i] c_{k}\left(t-i T-\tau_{k}\right)+v(t),
$$

where $A_{k}$ represents the received amplitude of the $k$ th user. The data bits $b_{k}[i]$ are independent identically distributed (i.i.d.) and assumed to be drawn from the finite alphabet $\{-1,+1\}$. The symbol period is denoted by $T$. The spreading (or signature) waveform of the $k$ th user $c_{k}(t)$ is $L_{c}$-dimensional and has unit energy property, i.e., $\left\|c_{k}\right\|^{2}=1$ and $\tau_{k} \in[0, T)$ are the relative offsets of the asynchronous signals at the receiver. The zero mean additive white Gaussian channel noise $v(t)$ has power spectral density $\sigma^{2}$ The amplitude $A_{k}$ and delay $\tau_{k}$ can be incorporated in the channel response $h_{k}(t)$ and the spreading code sequence $c(t)$ can be replaced with the discrete-time combined code-channel response

$$
g_{k}[n]=\sum_{i=0}^{L_{c}-1} c_{k}[i] h_{k}[n-i],
$$

where $c_{k}[i]$ is the $i$ th element of the code vector for the $k$ th user $\mathrm{c}_{k}=\left(c_{k}[0], \ldots, c\left[L_{c}-1\right]\right)^{T}$ and the received signal $r(t)$ in (1) can be written in the discrete-time vector form

$$
\mathbf{r}[n]=\sum_{k=1}^{K} \mathbf{r}_{k}[n]+\mathbf{v}[n]=\sum_{k=1}^{K} \mathbf{G}_{k} \mathbf{b}_{k}[n]+\mathbf{v}[n],
$$

where $\mathbf{G}_{k}$ is the combined code-channel response matrix of the $k$ th user and $b_{k}[n]=\left(b_{k}[n], b_{k}[n-1], \cdots, b_{k}\left[n-L_{b}+1\right]\right)^{T}$ 
and $\mathbf{v}[n]=\left(v[n], v[n-1], \cdots, v\left[n-L_{b}+1\right]\right)^{T}$ with $L_{b}=$ $\left\lceil\frac{L_{f}+L_{h}-1}{L_{\mathrm{c}}}\right\rceil$. Note that

$$
\mathbf{G}_{k}=\mathbf{C}_{k} \mathbf{H}_{k},
$$

where the code matrix $\mathbf{C}_{k}$ and the channel matrix $\mathbf{H}_{k}$ are

$$
\begin{gathered}
\mathbf{C}_{k}=\left(\begin{array}{ccc}
c_{k}\left[L_{c}-1\right] & & \\
\vdots & \ddots & \\
c_{k}[0] & & c_{k}\left[L_{c}-1\right] \\
& \ddots & \vdots \\
& & c_{k}[0]
\end{array}\right), \\
\mathbf{H}_{k}=\left(\begin{array}{llll}
\mathbf{h}_{k} & & & \\
& \mathbf{h}_{k} & & \\
& & \ddots & \\
& & & \mathbf{h}_{k}
\end{array}\right),
\end{gathered}
$$

where the channel response vector for the $k$ th user has length $L_{h}$, i.e., $\mathbf{h}_{k}=\left(h\left[L_{h}-1\right], \ldots, h[0]\right)^{T}$. The elements of the $L_{f} \times L_{b}$ combined code-channel matrix $\mathbf{G}_{k}$ are derived from (2):

$$
\mathbf{G}_{k}=\left(\begin{array}{ccc}
g_{k}\left(L_{c}-1\right) & \cdots & g_{k}\left(L_{b} L_{c}-1\right) \\
g_{k}\left(L_{c}-2\right) & \cdots & g_{k}\left(L_{b} L_{c}-2\right) \\
\vdots & & \vdots \\
g_{k}\left(L_{c}-L_{f}\right) & \cdots & g_{k}\left(L_{b} L_{c}-L_{f}\right)
\end{array}\right) .
$$

We can represent (3) as

$$
\mathbf{r}[n]=\mathcal{G} \mathbf{b}[n]+\mathbf{v}[n],
$$

where $\mathcal{G}=\left(\mathbf{G}_{1} \mathbf{G}_{2} \ldots \mathbf{G}_{K}\right)$ representing the overall combined code-channel matrix and $\mathbf{b}[n]=\left(\mathbf{b}_{1}^{T}[n], \mathbf{b}_{2}^{T}[n], \cdots, \mathbf{b}_{K}^{T}[n]\right)^{T}$ the overall data vector. Finally, we introduce the $L_{b} K \times 1$ combined code-channel-equalizer (system) response vector as

$$
\mathbf{q}[n]=\mathbf{w}^{T}[n] \mathcal{G},
$$

where $\mathrm{w}[n]$ is the $L_{f} \times 1$ weight vector of an equalizer at time $n$.

\section{COMBINED STRUCTURE OF CMOE AND CMA FOR RAKE RECEIVER}

Initialization is probably the most influential factor that controls the convergence behaviour of CMA [6], [7]. Therefore, to achieve reliable reception for a CMA receiver in near-far situations, an initialization technique which is robust to near-far environments is required. The CMOE criterion is preferable in interference suppression especially in severe near-far situations due to its decorrelating nature [5], [2]. We propose a novel initialization scheme for the CMA receivers in the RAKE receiver structure with the solution derived from the minimization of the CMOE criterion that satisfies the sufficient conditions stated in [6] and, with more extended results, in [7]

\subsection{Admissibility of CMOE as an initialization scheme for a CMA equalizer}

It has been shown in [7] that in multiuser systems the initial kurtosis condition of outputs is not the sole indicator that controls the CM-SG convergence as claimed in [6]. It is the SINR condition that is more restrictive and computing SINR is more appropriate when sample size is restricted to be small [7]. Therefore, in this paper we then concentrate on finding the initial estimator by the solution derived by minimizing the MOE criterion that satisfies the minimum requirement to the SINR condition. We define the minimum and maximum (normalized) kurtoses of the interference signals without the assumption of zero inter-symbol interference (ISI) as

$$
\kappa_{s}^{\min }=\min _{1 \leq k \leq K} \kappa_{s}^{(k)} \text { and } \kappa_{s}^{\max }=\max _{1 \leq k \leq K} \kappa_{s}^{(k)} .
$$

The minimum SINR at delay $\nu$ for non-super-Gaussian signals for the $k$ th user is verified to be [7]

$$
\operatorname{SINR}_{\min , \nu}^{(k)}=\frac{\sqrt{1+\rho_{\min }}}{2-\sqrt{1+\rho_{\min }}}, \quad \kappa_{s}^{\max } \leq \kappa_{g},
$$

where $\rho_{\min }=\frac{\kappa_{g}-\kappa_{g}^{\min }}{\kappa_{g}-\kappa_{g}^{(k)}}$ and $\rho_{\max }=\frac{\kappa_{g}-\kappa_{\alpha}^{\max }}{\kappa_{g}-\kappa_{g}^{(k)}}$ with $\kappa_{g}$ representing the (normalized) kurtosis of Gaussian signals. It has been shown that if $\operatorname{SINR}_{\nu}^{(k)}(y[n])>\operatorname{SINR}_{\min , \nu}^{(k)}$, the estimator will offer the estimates of the source $k$ with delay $\nu$.

Lemma 1: For the CM estimator $\mathbf{w}_{k}$ which is initialized by the weight vector $\mathrm{f}_{k}$ which minimizes the CMOE criterion at the SINR greater than the minimum SINR, i.e.,

$$
\mathbf{w}_{k}^{(i n i t)}:=\mathbf{f}_{k}[n] \text { s.t. } \operatorname{SINR}_{\nu}^{(k)}(y[n])>\operatorname{SINR}_{\min , \nu}^{(k)},
$$

then the $\mathrm{w}_{k}$ will preserve the estimates for the desired user $k$ at delay $\nu$.

\subsection{CMOE-initialized CMA RAKE receiver structure}

As in the case of the D-RAKE MOE receiver, the CMOE-CMA RAKE structure tries to collect the energies of the signals of the other paths. We introduce a constrained MOE initialization part for CMA. Firstly, we adopt the constraint proposed by Tsatsanis [2] which is shown to be

$$
\mathbf{f}_{k, l}^{T} \mathbf{C}_{k}=(0, \ldots, 0, \underset{\uparrow}{1}, 0, \ldots, 0) \triangleq \mathbf{1}_{l}^{T} .
$$

This corresponds to the $l$ th position of the rays. Denote the output energy of the $l$ th detector as

$$
J_{\mathrm{MOE}_{k}}^{(l)}=\mathbf{f}_{k, l}^{T} \mathbf{R f}_{k, l},
$$

where $\mathbf{R}=E\left\{\mathbf{r}[n] \mathbf{r}^{T}[n]\right\}$. We desire to minimize $J_{M O E}^{(l)}$ in order to suppress the energy of interference by

$$
\min _{\mathbf{f}_{h, l}} J_{\mathrm{MOE}_{k}}^{(l)} \text { s.t. } \quad \mathbf{f}_{k, l}^{T} \mathbf{C}_{k}=\mathbf{1}_{l}^{T} .
$$

By taking the instantaneous value of the autocorrelation matrix $\mathbf{R}$, the adaptation rule for the $l$ th CMOE detector of the $k$ th user becomes [1]

$$
\mathbf{f}_{k, l}[n+1]=\mathbf{f}_{k, l}[n]-\mu_{\mathrm{cmoe}_{l},} \boldsymbol{\Pi}_{\mathrm{C}_{\boldsymbol{b}}}^{1} \mathbf{r}[n] \mathbf{r}^{T}[n] \mathbf{f}_{k, l}[n],
$$

where $\mu_{\text {cmoc, } l}$ is the stepsize of the $l$ th detector and $\Pi_{\mathbf{C}_{h}}^{l}=I-$ $\mathbf{C}_{k}\left(\mathbf{C}_{k}^{T} \mathbf{C}_{k}\right)^{-1} \mathbf{C}_{k}^{T}$ denotes the projection matrix onto the nullspace of $\mathbf{C}_{k}$. The initialization of the lth detector is

$$
\mathbf{f}_{k, l}^{(i n i t)}=\mathbf{C}_{k}^{\dagger} \mathbf{1}_{l}
$$


where $\mathbf{C}_{k}^{\dagger}$ is the pseudo-inverse of $\mathbf{C}_{k}^{T}$. As shown in [2], the $l$ th detector in $\mathbf{C}_{k}$ whose delay corresponds to that of the dominan path can be identified by using the maximum MOE criterion after the convergence of all $L_{c}$ detectors. The estimated MOE of the $l$ th detector is

$$
\hat{J}_{\mathrm{MOE}_{k}}^{(l)}[n]=\frac{1}{N_{t}} \sum_{j=n-N_{t}+1}^{n} y_{k, l}^{2}[j]
$$

where $y_{k, l}[n]=\mathbf{f}_{k, l}^{T}[n] \mathbf{r}[n], \forall l \in\left\{1, \ldots, L_{c}\right\}$ denote the output signals of the $l$ th arm at time $n$ and $N_{t}$ is the sample size of the data symbols. We determine the first arm by that which gives the maximum output energy

$$
\mathbf{f}_{k, 1}[n]=\arg \left\{\max _{l} \hat{J}_{\mathrm{MOE}_{b}}^{(l)}[n]\right\} .
$$

The set of weight vectors of all arms of the RAKE structure can be constructed as $\left\{\mathbf{f}_{k, l}[n], \forall l \in\left\{1, \ldots L_{a}\right\}\right\}$ where $L_{a}$ denotes the number of arms and $L_{a}$ can be set to be much less than $L_{c}$ since the long delay paths usually correspond to small energies of the received signals. To guarantce convergence to the desired user solution as stated in Lemma 1 , we initialize the first arm $(l=1)$ of the $\mathrm{CM}$ equalizers with the weight vector of the first arm of the CMOE detectors at the first symbol $(\nu=1)$ with the minimum SINR. For a system with sub-Gaussian signals with none of the interference signals bearing kurtosis less than that of the desired user, the minimum $\operatorname{SINR}_{\min , \nu}$ is $3.8 \mathrm{~dB}$, i.e.,

$$
\mathbf{w}_{k, 1}^{(i n i t)}:=\mathbf{f}_{k, 1}\left[N_{s}\right] \text { s.t. } \operatorname{SINR}_{1}^{(k, 1)}\left(y_{k, 1}\left[N_{s}\right]\right)>3.8 \mathrm{~dB} \text {, }
$$

where $N_{s}$ is the time to adopt the solution derived by the minimization of the $\mathrm{CMOE}$ criterion to initialize the $\mathrm{CM}$ equalizer for the first arm. By CMA algorithm, for real signals, the criterion minimizes the following cost [8]

$$
J_{\text {CMA }}^{(k, l)}=E\left\{\left(z_{k, l}^{2}-\gamma_{k, l}\right)^{2}\right\},
$$

where $z_{k, l}=\mathbf{w}_{k, l}^{T} \mathbf{r}$ is the output symbol of the $l$ th equalizer for the $k$ th user and the dispersion constant $\gamma_{k, l}$ defines the gain of the lth CMA equalizer. The gain of each arm depends on the channel response at the corresponding delay, i.e., $\gamma_{k, l}=h_{k}^{2}[l]$. From the construction of the D-RAKE receiver, the outputs of the $l$ th arm are shown to be

$$
y_{k, l}[n]=h_{k}[l] b_{k}[n]+e_{k, l}[n],
$$

where $e_{k, l}[n]$ denotes the effective noise. This enables us to approximate the magnitude of the channel coefficient at the $l$ th delay. Since $E\left\{b_{k}^{2}\right\}=1$ and $e_{k, l}[n] \ll h_{k}[l] b_{x k}[n]$, we can approximate the gain $\gamma_{k, l}, \forall l \in\left\{1, \ldots, L_{a}\right\}$, by

$$
\gamma_{k, l} \approx \hat{J}_{\mathrm{MOE}}^{(k, l)}\left[N_{s}\right]=\frac{1}{N_{t}} \sum_{j=N_{s}-N_{t}}^{N_{s}} y_{k, l}^{2}[j]
$$

where $N_{t}$ is the sample size of the data symbols and $N_{s}$ is acheived in (20). The adaptation rule for the CM-RAKE receiver is

$$
\mathbf{w}_{k, l}[n+1]=\mathbf{w}_{k, l}[n]-\mu_{\mathrm{cma}, l} \mathbf{r}[n] z_{k, l}[n] e_{k, l}[n],
$$

where $\mu_{\mathrm{cms}, l}$ is the stepsize and $e_{k, l}[n]=z_{k, l}^{2}[n]-\gamma_{k, l}$ is the error signal for the $l$ th arm of the $k$ th CMA equalizer. After convergence, the arm outputs will be coherently combined to enhance the SINR by a coherent combiner which is essentially the principal eigenvector of the sample autocorrelation of the outputs of all arms $z_{k}[n]=\left(z_{k, 1}[n], \ldots, z_{k, L_{a}}[n]\right)^{T}$. The structure of the CMOE-CMA RAKE receiver is shown in Fig. 1.

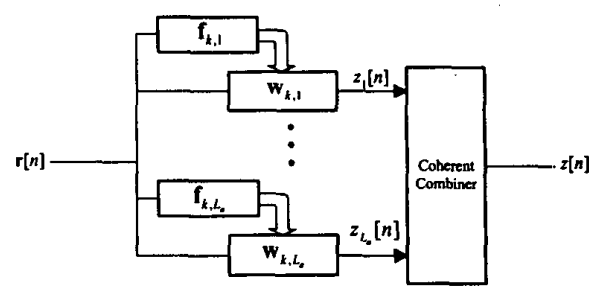

Fig. 1. The CMOE-CMA RAKE receiver.

\section{SIMULATIONS}

We considered a 10 -user $(K=10)$ symbol asynchronous system and we assumed without loss of generality that the first user was the user of interest with unity power. We used Gold codes with processing gain $L_{c}=31$ for the spreading codes of all users. User delays $\tau_{k}$ were uniformly distributed over $\left[0,3 T_{c}\right)$ and then kept fixed. The propagation channels are bandlimited with rootraised-cosine pulse shaping with excess bandwidth 0.2 . The number of multipath rays was four where the last three rays were uniformly distributed in delay over $\left[0,5 T_{c}\right)$ with standard deviation of 0.4. The channel length was $5 T_{c}$ and assumed to be the same for all users. The background noise was zero-mean AWGN with $\mathrm{SNR}=20 \mathrm{~dB}$ (referenced to the desired user). The performance measure was the average SINR in $\mathrm{dB}$ which is given by [5]

$$
\operatorname{SINR}_{a v}^{(1, l)}[n]=\frac{\sum_{r=1}^{R}\left[\mathbf{w}_{1, l}^{(r)^{T}}[n] \mathbf{r}_{1}^{(r)}[n]\right]^{2}}{\sum_{r=1}^{R}\left[\mathbf{w}_{1, l}^{(r)}[n]\left(\mathbf{r}^{(r)}[n]-\mathbf{r}_{1}^{(r)}[n]\right)\right]^{2}},
$$

where $\mathbf{r}_{1}^{(r)}[n]$ are the transmitted signals of the first user as described in (3) and $r$ denotes the index of a particular run. All SIR plots were averaged over 100 Monte-Carlo runs $(R=100)$. For all receivers, the number of arms was three $\left(L_{a}=3\right)$ and the receiver length was chosen to be twice that of the spreading gain $\left(2 L_{c}\right)$. We compare the SINR performances of the proposed CMOE-CMA RAKE receiver with the D-RAKE CMOE receiver [1] and the CM-RAKE receiver [3] in near-far environments. For a relatively high near-far level, $\frac{A_{1}^{2}}{A_{b}^{2}}=-10 \mathrm{~dB}, \forall k=$ $\{2, \ldots, 9\})$ as demonstrated in Fig. 2 , the proposed CMOE-CMA receiver is shown to exhibit the best SINR performance while the CMA-RAKE receiver performs better than the D-RAKE. Note that the CMA-RAKE receiver in [3] assumes exact knowledge of the correct timing of the desired user. If this is not the case, the kurtosis-based initialization scheme may be used as in [4]. However, a large number of samples is required to achieve reliable estimates of the kurtoses. The benefit of employing the RAKE structure is also demonstrated as the SINR performance of the CMOECMA RAKE receiver is improved from that achieved by the single (1st) arm CMOE-CMA receiver. In Fig. (3), it is shown that the SINR levels of the CMOE-CMA RAKE receiver is maintained over a wide range of the near-far situations. This confirms the nearfar resistance of the proposed receiver. The comparison of the system responses of each arm at initial state $\mathbf{q}_{\text {init }}^{(l)}$ and at steady-state (after convergence) $q_{\mathrm{ss}}^{(l)}$ at $\frac{A_{1}^{2}}{A_{\mathrm{t}}^{2}}=-10 \mathrm{~dB}$, are shown in Fig. 4. The corresponding initial SINRs for the lth arm are 7,6 and $5 \mathrm{~dB}$ for $l=1,2$ and 3 respectively. After all CMA equalizers con- 


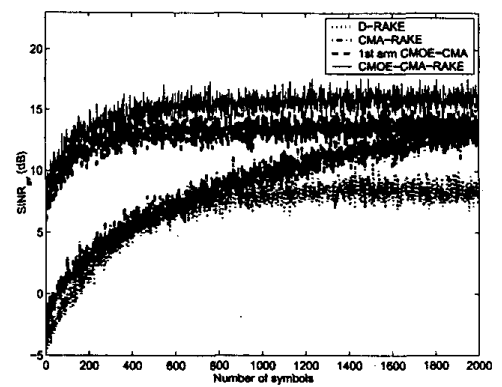

Fig. 2. The SINR performance of the CMOE-CMA-RAKE receiver compared with that of the D-RAKE receiver [1], the CMARAKE receiver [3] and the single (1st) arm CMOE-CMA receiver at $\frac{A_{1}^{2}}{A_{t}^{2}}=-10 \mathrm{~dB}$. Note the SINR improvement of the CMOECMA RAKE receiver over the single-arm CMOE-CMA receiver.

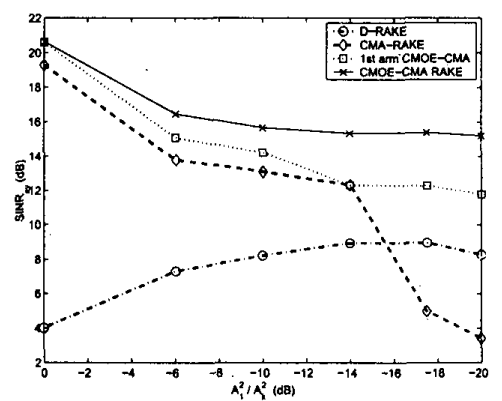

Fig. 3. Comaparison of the SINR performance at different nearfar levels. Note the near-far resistance of the CMOE-CMA RAKE receiver over a wide range of near-far levels.

verge, the steady-state system reponses $q_{\mathrm{ss}}^{(l)}$ are shown in Fig. 4 (b), (d), and (f). It is confirmed that the first user is preserved at the correct delay by the non-zero gains of $\mathbf{q}_{\mathrm{ss}}^{(l)}$ at $\nu=1$ while the interference is further suppressed by CMA.

\section{CONCLUSIONS}

The minimization of the CMOE criterion is adopted as an initialization scheme for a CMA-RAKE receiver for asynchronous CDMA systems. It is confirmed by simulations that the CMOECMA RAKE receiver is near-far resistant as long as the initialization satisfies the minimum SINR requirement. The RAKE receiver structure is used for combining the desired signal energies at different chip delays. The SINR performance is shown to be improved after coherently combining the outputs from all of the receiver arms.

\section{REFERENCES}

[1] H. Liu and K. Li, "A decorrelating RAKE receiver for CDMA communications over frequency-selective fading channels,"
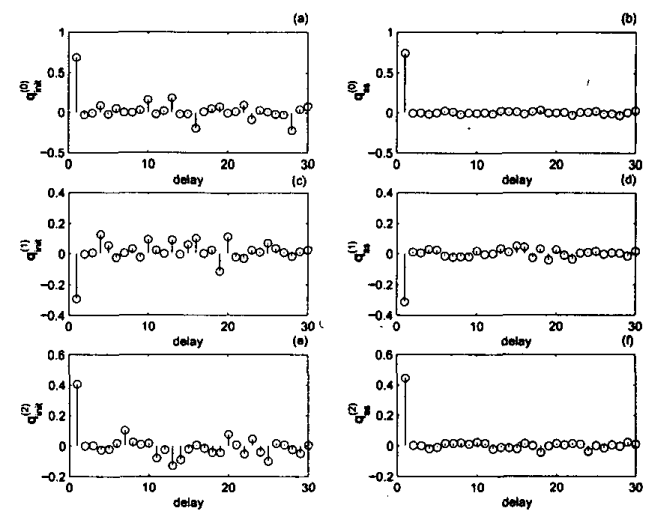

Fig. 4. Comparison of the initial and steady-state system responses of each arm at $\frac{A_{1}^{2}}{A_{b}^{2}}=-10 \mathrm{~dB}$. The initial system responses of each arm, $\mathrm{q}_{\text {init }}^{(l)}$, are shown in subfigure (a), (c) and (e) for $l=1,2$ and 3 respectively. After equalization, the steady-state system responses, $\mathrm{q}_{\mathrm{ss}}^{(l)}$, are shown in subfigure (b), (d) and (f).

IEEE Trans. Commun., vol. 47, no. 7, pp. 1036-1045, 1999.

[2] M. K. Tsatsanis, "Inverse filtering criteria for CDMA systems," IEEE Trans. Signal Processing, vol. 45, no. 1, pp. 102-112, 1997.

[3] M. Torlak and B. L. Evr.,As, "A self-recovering RAKE receiver for asynchronous CDMA systems," in Proc. Int. Conf. Acoustics, Speech and Signal Processing, Istanbul, Turkey, 2000, vol. 5, pp. 2869-2872.

[4] P. Schniter and C. R. Johnson Jr., "Minimum-entropy blind acquisition/equalization for uplink DS-CDMA," in Proc. Allerton Conf. on Communications, Control, and Computing, Monticello, IL, 1998, pp. 401-410.

[5] M. L. Honig, U. Madhow, and S. Verdú, "Blind adaptive multiuser detection," IEEE. Trans. Inform. Theory, vol. 41, pp. 944-966, 1995.

[6] Y. Li and Z. Ding, "Convergence analysis of finite length blind adaptive equalizers," IEEE Trans. Signal Processing, vol. 43, no. 9, pp. 2120-2129, 1995.

[7] P. Schniter and C. R. Johnson Jr., "SINR-based sufficient conditiions for CMA desired-user-lock," in Proc. IEEE WCNC, New Orleans, 1999, vol. 2, pp. 814-818.

[8] D. N. Godard, "Self-recovering equalization and carrier tracking in two-dimensional data communication systems," IEEE Trans. Commun., vol. 28, pp. 1867-1875, 1980. 